\title{
Physiological profile of Sri Lankan male long distance runners and sprinters competing at national level and university level; a descriptive study
}

\author{
Wijayasiri KDCU ${ }^{1,2}$, Wimalasekera $\mathrm{SW}^{3}$, Sivayogan $\mathrm{S}^{4}$, Waidyasekara $\mathrm{H}^{3}$, Ishikawa $\mathbf{Y}^{5}$ \\ ${ }^{\prime}$ Faculty of Graduate Studies, University of Sri Jayewardenepura, Nugegoda, Sri Lanka. \\ ${ }^{2}$ Sports and Exercise Medicine Unit, National Hospital of Sri Lanka, Colombo, Sri Lanka. \\ ${ }^{3}$ Department of Physiology, University of Sri Jayewardenepura, Nugegoda, Sri Lanka. \\ ${ }^{4}$ Department of Community Medicine, University of Sri Jayewardenepura, Nugegoda, Sri Lanka. \\ ${ }^{5}$ Cardiovascular Research Institute, Graduate School of Medicine, Yokohama City University, Yokohama, Japan.
}

\author{
Correspondence: Dr. K.D.C. Upendra Wijayasiri \\ e-mail: upendra.wija@gmail.com \\ (D) https://orcid.org/0000-0003-3678-8496 \\ Submitted on 23.04.2021 and accepted for publication on 07.05.2021
}

\begin{abstract}
Introduction: Despite regular training Sri Lankan runners do not perform well in international sports arena. The performance of a runner is determined by cardiopulmonary fitness level. However, little is known about the cardiopulmonary fitness levels of Sri Lankan runners. Thus, the present study was conducted to assess the status of the cardiopulmonary fitness of a group of Sri Lankan national level and university level male long distance runners and sprinters.
\end{abstract}

Methods: A descriptive cross-sectional study was conducted to determine the baseline data using interviewer administered questionnaire and clinical examination of 95 male runners [national level long distance runners $(\mathrm{n}=27)$, national level sprinters $(\mathrm{n}=27)$, university level long distance runners $(\mathrm{n}=20)$, university level sprinters $(\mathrm{n}=21)]$ and age and sex matched control subjects $(\mathrm{n}=41)$. Cardiopulmonary exercise fitness was assessed by a fully automated Cardiopulmonary Exercise Testing (CPET) machine.

Results: The CPET parameters such as $\mathrm{VO}_{2 \max }$, peak workload, exercise duration, heart rate at rest $\left(\mathrm{HR}_{\mathrm{res}}\right)$ and at peak $\left(\mathrm{HR}_{\text {peak }}\right)$, systolic blood pressure at rest $\left(\mathrm{SBP}_{\text {rest }}\right)$, and at peak $\left(\mathrm{SBP}_{\text {peak }}\right)$, diastolic blood pressure at rest $\left(\mathrm{DBP}_{\text {ress }}\right)$, and at peak $\left(\mathrm{DBP}_{\text {peak }}\right)$ amongst Sri Lankan national level runners indicated minimal improvement when compared to Sri Lankan university level runners. Further, most of the CPET parameters of Sri Lankan runners were lower when compared with those parameters of runners from the Asian region.

Conclusions and recommendations: Cardiopulmonary fitness of Sri Lankan long distance runners and sprinters is low. Thus, training protocols are needed to be modified in accordance with the baseline CPET parameters to attain optimal cardiovascular remodelling to enhance he performance of Sri Lankan runners.

Key words: Cardiopulmonary fitness, cardiopulmonary fitness assessment, running athletes, running performance. 


\section{Introduction}

The physical training and exercise promote functional and structural changes in cardiovascular system resulting in physiological modifications desirable for an athlete to give his/ her optimal performance. These changes are collectively known as cardiovascular remodelling. Cardiopulmonary exercise testing (CPET) is a widely accepted mode to determine cardiopulmonary response to training of athletes. Optimal cardiopulmonary fitness of an athlete indicates the integrated ability of the body to transport oxygen from the atmosphere to the mitochondria in muscles and remove the metabolic by-products from the active muscles during high intensity physical activity. Thus CPET parameters are dependent on a linked chain of processes that include pulmonary ventilation, diffusion of gases at the level of lungs and tissues, cardiac autonomic control, functions of the right and left ventricles (during both systole and diastole), the ability of the vasculature to accommodate and efficiently transport blood from the heart to active muscles and the ability of the muscle cells to receive and use the oxygen and nutrients delivered by the blood (1). Thus, CPET parameters comprehensively determine the function of the heart, the circulation and the respiratory system.

Maximal $\mathrm{O}_{2}$ consumption $\left(\mathrm{VO}_{2 \max }\right)$ is a main CPET parameter which comprehensively describes the cardiopulmonary fitness level and it can be used to measure the adaptations of the runners to the training. Most important factors that influence the individual differences of $\mathrm{VO}_{2 \max }$ are the mode of exercise and the trained state of the body, body composition, heredity, gender, and chronological age (2).

A high $\mathrm{VO}_{2 \max }$ is a prerequisite for success in longdistance running performance. Endurance training of male and female runners engaged in long distance running events such as $10,000 \mathrm{~m}$ and marathon events, increases $\mathrm{VO}_{2 \max }$ by $10 \%-25 \%$ compared to untrained sedentary people (3). The $\mathrm{VO}_{2 \max }$ of some professional marathoners have been reported as nearly as twice of the $\mathrm{VO}_{2 \max }$ of sedentary individuals (4). In contrast to endurance training, sprint runners will increase the $\mathrm{Vo}_{2 \max }$ by $5 \%-12 \%$ with training, compared to untrained people irrespective of gender (5).
Training results in an enhanced parasympathetic tone over the sympathetic activity on the heart. Thus, regular training results in adaptation of the heart; a major manifestation of the cardiac regulatory mechanisms (6). This effect is more pronounced in athletes engaged in endurance training as indicated by higher reduction in $\mathrm{HR}_{\text {rest }}$ as much as $43 \%$ when compared to untrained individuals (7). Well trained elite athletes with high endurance have significantly lower peak heart rates than the untrained individuals at a given $\mathrm{VO}_{2 \max }(8)$.

The blood pressure response may be detrimental to the exercise performance of athletes (9). As reported in literature, professional endurance runners have significantly lower resting blood pressure than normal people. Studies report a reduction in mean $\mathrm{SBP}_{\text {rest }}$ by $05 \mathrm{mmHg}$ and a reduction in mean $\mathrm{DBP}_{\text {rest }}$ by $03 \mathrm{mmHg}$ after endurance training (10).

Considering the above background, the present study was conducted to assess cardiopulmonary fitness parameters amongst Sri Lankan athletes engaged in long distance running events $(10,000 \mathrm{~m}$ and marathon) and sprinters (engaged in $100 \mathrm{~m}$ and $200 \mathrm{~m}$ running) in comparison with age, gender and height-matched controls. Since there is a paucity of CPET data on Sri Lankan runners, these values would be useful to guide training schedules of Sri Lankan runners to enhance their performance to reach international standards.

\section{Methods}

The study group consisted of 95 male runners; National Level Long Distance Runners (NLDR, $\mathrm{n}=27$ ), National Level Sprinters (NLS, $\mathrm{n}=20$ ), University Level Long Distance Runners (ULDR, $\mathrm{n}=27$ ), University Level Sprinters (USR, $\mathrm{n}=21$ ). National level runners competing at national and international level and university runners competing at Sri Lanka University Games were recruited into the study. Age, sex and height matched sedentary adults who were not attending in regular sports training were studied as control subjects $(n=41)$. Those who had past or present history of musculoskeletal injuries or chronic non communicable diseases were excluded from the study. 
All participants gave informed written consent. They were assessed using an interviewer administered questionnaire to obtain baseline data and a full clinical examination including resting ECG to confirm general health before start the study. Height and weight were measured for the calculation of body mass index (BMI). Systolic and diastolic blood pressure were measured at rest and just after exercise.

Prior to conducting each test, the CPET machine was calibrated by using the calibration cylinder according manufacturer's guidelines. CPET for the runners and controls was carried out by using continuous incremental protocol $(30 \mathrm{~W} /$ minute) up to exhaustion (11) with electromagnetically braked cycle ergometer (COSMED, Italy). Real time gas analysis was conducted using a fully automated CPET machine (Fitmate $^{\mathrm{TM}}$, COSMED, Italy). The parameters assessed were $\mathrm{VO}_{2 \max }$ ( $\mathrm{ml} / \mathrm{kg} / \mathrm{min}$ ), exercise duration (minutes), exercise capacity (Metabolic Equivalents; METs), work load $(\mathrm{W})$, heart rate at rest $\left(\mathrm{HR}_{\text {rest }}\right)$ and at peak $\left(\mathrm{HR}_{\text {peak }}\right)$ (beats. $\left.\min ^{-1}\right)$, systolic blood pressure at rest $\left(\mathrm{SBP}_{\text {rest }}\right)$ and at peak $\left(\mathrm{SBP}_{\text {peak }}\right)(\mathrm{mmHg})$, diastolic blood pressure at rest $\left(\mathrm{DBP}_{\text {ress }}\right)$ and at peak, $\left(\mathrm{DBP}_{\text {peak }}\right)$ (mmHg).

Ethical approval was granted for the study by the Ethics Review Committee of Faculty of Medical Sciences, University of Sri Jayewardenepura.

\section{Statistical analysis}

Data were analysed by using the Statistical Package for the Social Sciences (SPSS) version 20. Data were compared using ANOVA and Student's t-test.

\section{Results}

The mean values of age, height, weight, BMI and duration of training of NLDR, ULDR and controls are summarised in Table 1. The mean weight and mean BMI of male controls was significantly higher than the mean weight and mean BMI of NLDR and ULDR $(p<0.05)$. Even though the duration of training between NLDR and ULDR was similar $(p>0.05)$, NLDR were engaged in significantly higher number of training hours per week than the $\operatorname{ULDR}(p<0.05)$.

The mean age, height, weight, BMI and duration of training of NSR, USR and controls are summarised in Table 2. The mean age, height, weight, BMI and duration of training were not significantly different between NSR, USR and controls $(p>0.05)$. The mean training hours per week of NSR was significantly higher than the USR $(p<0.05)$.

CPET parameters of national level runners, university level runners and controls are summarised in Tables 3 and 4.

Table 1: Age, anthropometry and training data of national level and university level long distance runners and controls

\begin{tabular}{lccc}
\hline Baseline Characteristics & $\begin{array}{c}\text { NLDR }(\mathbf{n}=\mathbf{2 7}) \\
(\text { Mean } \pm \text { SD) }\end{array}$ & $\begin{array}{c}\text { ULDR }(\mathbf{n}=\mathbf{2 7}) \\
(\text { Mean } \pm \text { SD) }\end{array}$ & $\begin{array}{c}\text { Controls }(\mathbf{n}=\mathbf{4 1}) \\
(\text { Mean } \pm \text { SD) }\end{array}$ \\
\hline Age (years) & $23.7 \pm 4.9$ & $22.8 \pm 1.3$ & $23.0 \pm 1.4$ \\
Height $(\mathrm{cm})$ & $168.0 \pm 5.0$ & $169.0 \pm 6.3$ & $169.6 \pm 6.8$ \\
Weight $(\mathrm{kg})$ & $53.0 \pm 3.0^{*}$ ? & $55.4 \pm 7.1^{?}$ & $64.0 \pm 13.7$ \\
Body Mass Index $\left(\mathrm{kg} / \mathrm{m}^{2}\right)$ & $18.7 \pm 1.0^{* ?}$ & $19.2 \pm 2.13^{?}$ & $22.0 \pm 3.8$ \\
Training hours per week (hours) & $16.8 \pm 5.4^{\mu}$ & $9.4 \pm 3.0$ & -- \\
Duration of training (years) & $5.0 \pm 3.4$ & $6.3 \pm 4.1$ & -- \\
\hline
\end{tabular}

ANOVA test and Student's t-test used to compare the means

$\mu$ - Significantly higher than university runners; * - Significantly lower than university runners; ? - Significantly lower than the controls NDLR - National Level Long Distance Runners; ULDR - University Level Long Distance Runners 
Table 2: Age, anthropometry and training data of national level and university level sprinters and controls

\begin{tabular}{lccc}
\hline Baseline characteristics & $\begin{array}{l}\text { NSR } \\
(\mathbf{n}=\mathbf{2 0}) \\
(\mathbf{M e a n} \pm \mathbf{S D})\end{array}$ & $\begin{array}{l}\text { USR } \\
(\mathbf{n}=\mathbf{2 1}) \\
(\mathbf{M e a n} \pm \mathbf{S D})\end{array}$ & $\begin{array}{l}\text { Control } \\
(\mathbf{n}=\mathbf{4 1}) \\
(\text { Mean } \pm \text { SD) }\end{array}$ \\
\hline Age (years) & $23.8 \pm 3.2$ & $22.5 \pm 1.5$ & $23.0 \pm 1.4$ \\
Height $(\mathrm{cm})$ & $171.6 \pm 6.5$ & $173.8 \pm 5.3$ & $169.6 \pm 6.8$ \\
Weight $(\mathrm{kg})$ & $65.1 \pm 9.6$ & $65.0 \pm 13.1$ & $64.0 \pm 13.7$ \\
Body Mass Index $\left(\mathrm{kg} / \mathrm{m}^{2}\right)$ & $21.8 \pm 2.2$ & $21.8 \pm 4.2$ & $22.0 \pm 3.8$ \\
Training hours per week (hours) & $20.9 \pm 7.7^{\mu}$ & $8.9 \pm 4.2$ & -- \\
Duration of training (years) & $6.9 \pm 3.3$ & $5.9 \pm 3.6$ & -- \\
\hline
\end{tabular}

ANOVA test and Student's t-test used to compare the means

$\mu$ - Significantly higher than university runners; NSR - National Sprint Runners; USR - University Sprint Runners

Table 3: Cardiopulmonary fitness parameters of national level long-distance runners, university level long distance runners and controls

\begin{tabular}{|c|c|c|c|}
\hline Parameter & $\begin{array}{l}\text { NLDR } \\
(n=27) \\
\text { Mean } \pm \text { SD }\end{array}$ & $\begin{array}{l}\text { ULDR } \\
(n=27) \\
\text { Mean } \pm \text { SD }\end{array}$ & $\begin{array}{l}\text { Controls } \\
(n=41) \\
\text { Mean } \pm \text { SD }\end{array}$ \\
\hline $\mathrm{VO}_{2 \max }(\mathrm{ml} / \mathrm{kg} / \mathrm{min})$ & $58.2 \pm 9.4^{\mu \pi}$ & $50.6 \pm 7.4^{\pi}$ & $37.4 \pm 6.5$ \\
\hline METs & $16.6 \pm 2.6^{\mu \pi}$ & $14.4 \pm 2.1^{\pi}$ & $10.9 \pm 2.1$ \\
\hline Exercise Duration (min.) & $12.6 \pm 2.0^{\mu \pi}$ & $11.1 \pm 1.2 \rrbracket$ & $9.5 \pm 1.1$ \\
\hline Work load (W) & $302.1 \pm 79.4^{\mu \pi}$ & $259.2 \pm 39.5$ & $223.8 \pm 51.6$ \\
\hline $\mathrm{HR}_{\text {rest }}$ (beats/min) & $59.5 \pm 12.9^{* ?}$ & $68.0 \pm 10.2^{?}$ & $78.6 \pm 21.4$ \\
\hline $\mathrm{HR}_{\text {peak }}($ beats/min) & $165.8 \pm 12.1$ & $169.7 \pm 13.9$ & $164.4 \pm 19.7$ \\
\hline $\mathrm{SBP}_{\text {rest }}(\mathrm{mm} \mathrm{Hg})$ & $120.0 \pm 8.97$ & $120.0 \pm 10.76$ & $123.57 \pm 6.98$ \\
\hline $\mathrm{SBP}_{\text {peak }}(\mathrm{mm} \mathrm{Hg})$ & $158.46 \pm 13.48^{\mu \Phi}$ & $154.0 \pm 8.94 \pi$ & $144.39 \pm 8.38$ \\
\hline $\mathrm{DBP}_{\text {rest }}(\mathrm{mm} \mathrm{Hg})$ & $70.0 \pm 6.43^{?}$ & $70.0 \pm 8.32^{?}$ & $80.0 \pm 3.10$ \\
\hline $\mathrm{DBP}_{\text {peak }}(\mathrm{mm} \mathrm{Hg})$ & $85.12 \pm 5.06$ & $83.0 \pm 4.70$ & $85.85 \pm 4.98$ \\
\hline
\end{tabular}

ANOVA test used to compare the means

$\mu$ - Significantly higher than university runners; - Significantly higher than the controls; * - Significantly lower than university runners;

? - Significantly lower than controls; NDLR - National Level Long Distance Runners; ULDR - University Level Long Distance Runners 
Table 4: Cardiopulmonary fitness parameters of national level sprinters, university level sprinters and controls

\begin{tabular}{|c|c|c|c|}
\hline Parameter & $\begin{array}{l}\text { NSR } \\
(n=20) \\
\text { Mean } \pm \text { SD }\end{array}$ & $\begin{array}{l}\text { USR } \\
(n=21) \\
\text { Mean } \pm \text { SD }\end{array}$ & $\begin{array}{l}\begin{array}{l}\text { Controls } \\
(n=41)\end{array} \\
\text { Mean } \pm \text { SD }\end{array}$ \\
\hline $\mathrm{VO}_{2 \max }(\mathrm{ml} / \mathrm{kg} / \mathrm{min})$ & $47.6 \pm 8.4^{\pi}$ & $45.1 \pm 6.4^{\pi}$ & $37.4 \pm 6.5$ \\
\hline METs & $13.6 \pm 2.4^{\natural}$ & $12.8 \pm 1.8$ & $10.9 \pm 2.1$ \\
\hline Exercise Duration (min.) & $12.8 \pm 3.3^{\mu ף}$ & $10.9 \pm 1.3$ & $9.5 \pm 1.1$ \\
\hline Work load (W) & $285.6 \pm 78.1^{\mu \pi}$ & $269.2 \pm 36.0^{\oplus}$ & $223.8 \pm 51.6$ \\
\hline $\mathrm{HR}_{\text {rest }}$ (beats/min) & $66.0 \pm 10.3^{*} ?$ & $75.57 \pm 15.2$ & $78.67 \pm 21.4$ \\
\hline $\mathrm{HR}_{\text {peak }}($ beats/min) & $171.3 \pm 12.5^{\pi}$ & $172.2 \pm 11.6^{\pi}$ & $164.4 \pm 19.7$ \\
\hline $\mathrm{SBP}_{\text {rest }}(\mathrm{mm} \mathrm{Hg})$ & $123.31 \pm 4.97$ & $120.0 \pm 14.76$ & $123.57 \pm 6.98$ \\
\hline $\mathrm{SBP}_{\text {peak }}(\mathrm{mmHg})$ & $157.50 \pm 14.52^{\mu \pi}$ & $151.0 \pm 12.93$ & $144.39 \pm 8.38$ \\
\hline $\mathrm{DBP}_{\text {rest }}(\mathrm{mmHg})$ & $70.40 \pm 2.00^{?}$ & $70.0 \pm 6.97^{?}$ & $80.0 \pm 3.10$ \\
\hline $\mathrm{DBP}_{\text {peak }}(\mathrm{mmHg})$ & $85.60 \pm 5.06^{\mu}$ & $81.5 \pm 3.66^{?}$ & $85.85 \pm 4.98$ \\
\hline
\end{tabular}

ANOVA test used to compare the means

$\mu$ - Significantly higher than university runners; $\uparrow$ - Significantly higher than the controls; * - Significantly lower than university runners;

? - significantly lower than controls; NSR - National Sprint Runners; USR - University Sprint Runners

\section{Cardiopulmonary fitness parameters}

The mean $\mathrm{VO}_{2 \max }$, METs (functional capacity), exercise duration on cycle ergometer and work load of NLDR were significantly higher when compared with ULDR and control subjects $(p<0.05)$. ULDR had significantly higher $\mathrm{VO}_{2 \max }$, METs and duration of exercise in comparison to controls $(p<0.05)$. $\mathrm{HR}_{\text {rest }}$ was significantly lower amongst NLDR and ULDR than controls. $\mathrm{HR}_{\text {peak }}$ was not significantly different between runners and controls $(p>0.05)$. $\mathrm{SBP}_{\text {peak }}$ of NLDR and ULDR were significantly higher than that of the controls $(p<0.05)$. NLDR and ULDR had significantly lower $\mathrm{DBP}_{\text {rest }}$ compared to controls $(p<0.05)$.

The NSR had significantly higher exercise duration on cycle ergometer, work load than USR and controls $(p<0.05)$. However, the $\mathrm{VO}_{\text {max }}$ level and METs of NLDR had no significant difference when compared with USR. $\mathrm{HR}_{\text {rest }}$ and $\mathrm{HR}_{\text {peak }}$ of NSR and USR were significantly lower than the controls $(p<0.05)$. The mean $\mathrm{SBP}_{\text {peak }}$ of NSR and USR were significantly higher than the controls $(p<0.05)$. The mean $\mathrm{DBP}_{\text {rest }}$ of NSR and USR were significantly lower than the controls $(p<0.05)$. The mean $\mathrm{DBP}_{\text {peak }}$ of NSR and controls were significantly higher than the USR $(p<0.05)$.

\section{Discussion}

The purpose of this study was to determine the current status of CPET parameters of NLDR and NSR in comparison with ULDR, USR and controls. To the best of the authors' knowledge this is the first study conducted in Sri Lanka to assess the CPET parameters of national and university level runners using real time gas analysis.

In the present study, the age and the height were found to be similar between NLDR, NSR, ULDR, USR and control subjects. NLDR were found to 
have the lowest body weight and BMI amongst national level runners, university level runners and controls. This may be due to the engagement of NLDR in regular endurance training than the other runners. The distribution of age, height, weight and BMI of Sri Lankan NLDR and NSR were found to be similar to available data of international long distance runners in the region (12).

In the present study, $\mathrm{VO}_{2 \max }$ of NLDR was lower than the international long distance runners in the region (12). Deficiencies of the endurance training programs including unavailability of high-altitude training may be a reason for the lower $\mathrm{VO}_{2 \max }$ level of NLDR. However, the mean $\mathrm{VO}_{2 \max }$ of NLDR was significantly higher when compared to the $\mathrm{VO}_{2 \max }$ of ULDR and the control subjects.

In the present study, NLDR had lower peak work load at the point of exhaustion during the CPET assessment compared to previously reported data of Asian runners (13). However, the peak work load of NLDR was significantly increased compared to the university counterparts.

In the present study, as expected, higher $\mathrm{VO}_{2 \max }$ level was observed in NSR and USR when compared to controls. The reason for the higher levels of $\mathrm{VO}_{2 \max }$ amongst NSR and USR than controls may be the lower cardiopulmonary fitness levels of physically less active controls.

Sri Lankan NSR had achieved the $\mathrm{VO}_{2 \max }$ on par with the data of international sprinters in the region reported in the literature $(12,14)$. However, the USR and NSR also had similar level of $\mathrm{VO}_{2 \max }$.

The NLDR and NSR had significantly higher exercise duration on cycle ergometer, exercise capacity and peak work load along with the higher $\mathrm{VO}_{2 \max }$ when compared to ULDR and USR. When an athlete is engaged in regular training on selected groups of muscles, such as leg muscles for a longer duration of time it will cause an increase of $\mathrm{VO}_{2 \max }$ level and psychological tolerance to fatigue (15). Even though the national runners and the university runners were engaged in technical sports training for a similar duration; NLDR and NSR were engaged in significantly higher training hours per week than the university counterparts. The higher tolerance to fatigue achieved through longer training may be the reason for the significantly higher exercise duration on cycle ergometer, exercise capacity and peak work load of national runners than the University runners. However, the peak work load of NSR is far below than the peak work load of elite Asian sprinters (16). As explained in literature, there is a strong positive correlation of peak power output and running speed of elite sprinters (17). As depicted by the present study, lower peak work load may be a reason for poor performance of NSR and USR.

NLDR had the lowest $H_{\text {rest }}$ whereas the NSR and had the highest $\mathrm{HR}_{\text {peak }}$ amongst the national runners, University runners and the controls. Earlier studies have reported that professional endurance runners had significantly lower resting blood pressure $\left(\mathrm{SBP}_{\text {rest }}\right.$ and $\left.\mathrm{DBP}_{\text {ress }}\right)$ than other people (10). In the present study, an isolated reduction of $\mathrm{DBP}_{\text {rest }}$ was observed amongst national and university runners which is in accordance with the available data of Asian elite long distance runners (18). However, no difference was observed of mean $\mathrm{SBP}_{\text {rest }}$ amongst the national and university runners when compared to the controls. Significantly higher mean $\mathrm{SBP}_{\text {peak }}$ was observed amongst national runners when compared with the university counterparts and the control subjects. This may be due to the higher exercise level and the higher tolerance of fatigue amongst national runners gained through comparatively longer duration of training.

The results of the present study indicate that sub optimal remodelling of the cardiovascular system had been attained by NLDR and NSR irrespective of the rigorous training when compared to university counterparts. However, as depicted by lower $\mathrm{HR}_{\text {rest }}$ and $\mathrm{DBP}_{\text {rest }}$ of NLDR, the parasympathetic effect of training was pronounced amongst long distance runners when compared to sprinters and controls.

\section{Conclusions}

The present study concludes that lower levels of cardiopulmonary fitness parameters such as $\mathrm{VO}_{2 \max }$ and peak power output of Sri Lankan national long distance runners may be detrimental to optimal performance at international arena. Although Sri Lankan sprinters achieved similar $\mathrm{VO}_{2 \max }$ as reported amongst international sprinters, lower peak power output is a major limiting factor 
of performance. Suboptimal remodelling of the cardiovascular system is the main reason for the lower level of cardiopulmonary fitness of Sri Lankan long distance runners and sprinters. Training protocols are known to contribute to cardiovascular remodelling. Thus, training protocols need to be modified in accordance with the base line CPET parameters to attain optimal cardiovascular remodelling, and thus enhance performance.

\section{References}

1. Ross R, Blair SN, Arena R, Church TS, Després JP, Franklin BA, Haskell WL, Kaminsky LA, Levine BD, Lavie CJ, Myers J. Importance of assessing cardiorespiratory fitness in clinical practice: a case for fitness as a clinical vital sign: a scientific statement from the American Heart Association. Circulation. 2016 Dec 13; 134(24): e653-699.

2. Chatterjee S, Chatterjee P, Bandhopadhyay A. Cardiorespiratory fitness of obese boys. Indian Journal of Physiology and Pharmacology. 2005; 49(3): 353-357.

3. Mcardle WD, Katch FI, Katch VL. The pulmonary system and Physical activity. Essentials of Exercise Physiology. Fifth edition ed. two commerce square, 2001, Market place, Philadelphia, PA: Wolters Kluwer; 2016. p.251-284.

4. Swanwick E, Matthews M. Energy Systems: A New Look at Aerobic Metabolism in Stressful Exercise. MOJ Sports Med. 2018; 2(1): 00039.

5. Vollaard N, Metcalfe R. Williams S. Effect of number of sprints in a SIT session on change in $\mathrm{VO}_{2 \max }$ : a metaanalysis. Medicine and Science in Sports and Exercise. 2017; 49(6): 1147-1156.

6. Pierpont GL, Stolpman DR, Gornick CC. Heart rate recovery post-exercise as an index of parasympathetic activity. Journal of the Autonomic Nervous System. 2000 May 12; 80(3): 169-174.

7. Plews DJ, Laursen PB, Stanley J, Kilding AE, Buchheit M. Training adaptation and heart rate variability in elite endurance athletes: opening the door to effective monitoring. Sports Medicine. 2013 Sep; 43(9): 773-81.

8. McArdle WD, Katch FI, Katch VL. Training the Anaerobic and Aerobic energy systems. Essential of Exercise Physiology. Fifth edition. Baltiomre: Wolters Kluwer; 2016. p.403-432.
9. Riebe D, Ehrman JK, Liguori G, Magal M. Medicine ACoS. ACSM's guidelines for exercise testing and prescription: Wolters Kluwer; 2018.

10. Punia S, Kulandaivelan S, Singh V, Punia V. Effect of aerobic exercise training on blood pressure in Indians: Systematic Review. International Journal of Chronic Diseases. 2016; 2016: 1370148

11. Bentley DJ, Newell J, Bishop D. Incremental exercise test design and analysis. Sports Medicine. 2007 Jul; 37(7): 575-586.

12. Kelhar GK, Subhadra M, Chengappa RK. Body composition, haematological status and aerobic capacity of competitive Indian sportsmen. Indian Journal of Nutrition and Dietetics. 2008; 45(9): 379-387.

13. San-Millan I, Brooks GA. Assessment of Metabolic Flexibility by Means of Measuring Blood Lactate, Fat, and Carbohydrate Oxidation Responses to Exercise in Professional Endurance Athletes and Less-Fit Individuals. Sports Medicine. 2018; 48(2): 467-479.

14. Gantois P, Aidar FJ, De Matos DG, De Souza RF, Da Silva LM, De Castro KR, De Medeiros RC, Cabral BG. Repeated sprints and the relationship with anaerobic and aerobic fitness of basketball athletes. Journal of Physical Education and Sport. 2017 Jun 1; 17(2): 910.

15. Ghosh AK. Anaerobic threshold: its concept and role in endurance sport. The Malaysian Journal of Medical Sciences: MJMS. 2004 Jan; 11(1): 24-36.

16. Suzuki Y, Ito O, Mukai N, Takahashi H, Takamatsu K. High level of skeletal muscle carnosine contributes to the latter half of exercise performance during 30-s maximal cycle ergometer sprinting. The Japanese Journal of Physiology. 2002; 52(2): 199-205.

17. Morin JB, Bourdin M, Edouard P, Peyrot N, Samozino P, Lacour JR. Mechanical determinants of $100-\mathrm{m}$ sprint running performance. European Journal of Applied Physiology. 2012Nov; 112(11): 3921-30.

18. Hulke SM, Vaidya YP, Ratta AR. Effects of sixteen weeks exercise training on left ventricular dimensions and function in young athletes. National Journal of Physiology, Pharmacy and Pharmacology. 2012 Jul 1; 2(2): 152-158. 